\title{
Predicting the Aoki Phase using the Chiral Lagrangian*
}

\author{
Stephen Sharpe ${ }^{a}$ and Robert L. Singleton Jr. ${ }^{\text {a }}$

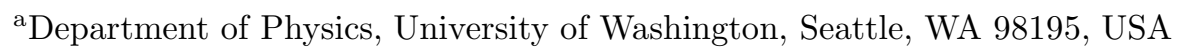

\begin{abstract}
This work is concerned with the phase diagram of Wilson fermions in the mass and coupling constant plane for two-flavor (unquenched) QCD. We show that as the continuum limit is approached, one can study the lattice theory using the continuum chiral Lagrangian, supplemented by additional terms proportional to powers of the lattice spacing. We find two possible phase structures at non-zero lattice spacing: (1) There is an Aoki phase of spontaneously broken flavor and parity, with two massless Goldstone-pions, and a width $\Delta m_{0} \sim a^{3} ;(2)$ There is no spontaneous symmetry breaking, and all three pions have equal mass of order $a$. Present numerical simulations suggest that the former option is realized.
\end{abstract}

Some time ago, Aoki proposed that lattice QCD with Wilson fermions has a phase in which flavor and parity are spontaneously broken, thereby providing a dynamical explanation for the presence of massless pions at non-zero lattice spacing[1]. His proposed phase diagram is shown in Fig. 1. This suggestion has been studied using analytical and numerical methods, and somewhat contradictory conclusions have been drawn'. We have recently shown that one can analyze the phase structure analytically close to the continuum limit[2]. In this paper we summarize the salient points of our work

We consider lattice QCD with two degenerate Wilson fermions. The action is invariant under flavor $S U(2)$ and parity, but explicitly breaks all axial symmetries for any value of the bare mass. Aoki's proposal is that the condensate $\left\langle\bar{\psi} i \gamma_{5} \sigma_{3} \psi\right\rangle$ is non-zero in region $B$ (with $\sigma_{3}$ acting in flavor space), thus breaking the flavor symmetry to a $U(1)$ subgroup and producing two massless Goldstone pions throughout $B$.

To investigate this suggestion analytically, we first write down an effective continuum description of the lattice theory valid close to the continuum limit, and then examine the long distance behavior of the effective theory using standard chiral Lagrangian techniques. The effective continuum theory can be described by a Lagrangian

\footnotetext{
*Supported by DOE contract DE-FG03-96ER40956. ${ }^{\dagger}$ Speaker.

${ }^{3}$ For detailed references to earlier work see Ref. [2]

${ }^{4} \mathrm{M}$. Creutz had previously performed a similar analysis based on the linear sigma model[3].
}

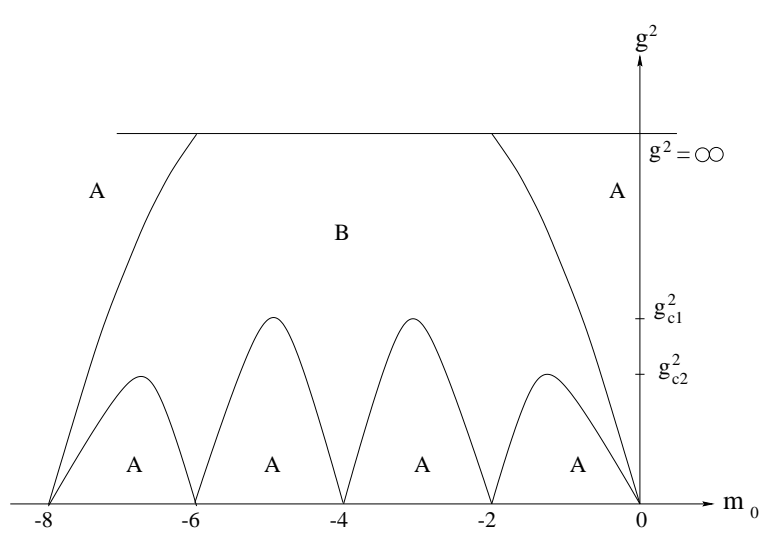

Figure 1. The phase diagram proposed by Aoki: $g$ is the gauge coupling and $m_{0}$ the dimensionless bare quark-mass. The continuum-like unbroken phases are labeled $A$, and the flavor and parity broken phase $B$. The continuum limit of particular interest is that at $m_{0}=0, g=0$.

in which the usual terms have been supplemented by higher dimensional operators proportional to powers of the lattice spacing [4]. These additional pieces are constrained only by the symmetries of the lattice action, which means that they need not respect chiral symmetry, and their enumeration is identical to that carried out as part of the improvement program [5]. As will become apparent shortly, we are interested in physical masses of order the lattice spacing, i.e. $m \sim a \Lambda^{2}$, with $\Lambda$ an abbreviation for $\Lambda_{\mathrm{QCD}}$. In this mass regime, with a suitable choice of quark variables, the ef- 
fective continuum description of the lattice theory can be written[2]

$\mathcal{L}_{\text {eff }}=\mathcal{L}_{\mathrm{g}}+\bar{\psi}(\not D+m) \psi+b_{1} a \bar{\psi} i \sigma_{\mu \nu} F_{\mu \nu} \psi$,

where $\mathcal{L}_{\mathrm{g}}$ is the gluon Lagrangian, and we have dropped terms of $\mathcal{O}\left(a^{2}\right)$. The dimensionless parameter $b_{1} \sim 1$ is a function of the coupling $g$, while the physical mass is $m \sim\left(m_{0}-\tilde{m}_{c}(g)\right) / a$, with $m_{0}$ the (dimensionless) bare mass and $\tilde{m}_{c}$ a critical mass.5 Both the mass term and the Pauli term in (i1) break chiral symmetry. A key point in the following is that when we adjust the bare mass at fixed $g$ (and thus fixed $a$ ) we change the relative importance of these two terms.

The next step is to write a generalization of the continuum chiral Lagrangian that includes the effects of the Pauli term. Without either the mass term or the Pauli term, the theory is invariant under $S U(2)_{L} \times S U(2)_{R}$ chiral rotations, and its low momentum dynamics is described by the chiral Lagrangian

$\mathcal{L}_{\chi}=\frac{f_{\pi}^{2}}{4} \operatorname{Tr}\left(\partial^{\mu} \Sigma^{\dagger} \partial_{\mu} \Sigma\right)$

Here $\Sigma$ is an $S U(2)$ matrix-valued field that transforms under the chiral group as $\Sigma \rightarrow L \Sigma R^{\dagger}$, with $L$ are $R$ being independent $S U(2)$ rotations. Its vacuum expectation value, $\Sigma_{0}=\langle\Sigma\rangle$, breaks the chiral symmetry down to an $S U(2)$ subgroup. The fluctuations around $\Sigma_{0}$ correspond to the Goldstone-bosons,

$\Sigma=\Sigma_{0} \exp \left\{i \sum_{a=1}^{3} \pi_{a} \sigma_{a} / f_{\pi}\right\}$.

The mass term explicitly breaks the underlying chiral symmetry, and a standard spurion analysis gives, to second order in $m$, the potential energy

$\mathcal{V}_{\chi}=-\frac{c_{1}}{4} \operatorname{Tr}\left(\Sigma+\Sigma^{\dagger}\right)+\frac{c_{2}}{16}\left\{\operatorname{Tr}\left(\Sigma+\Sigma^{\dagger}\right)\right\}^{2}$

with $c_{1} \sim m \Lambda^{3}$ and $c_{2} \sim m^{2} \Lambda^{2}$. Since the Pauli term transforms under chiral rotations as does the mass term, and as it is proportional

${ }^{5}$ The symbol $\sim$ means that we are not attempting to keep track of renormalization factors of order unity.

${ }^{6}$ Kinetic energy corrections to $\mathcal{L}_{\chi}$ need not be considered since we are only interested in the vacuum state. to a single power of the lattice spacing, its effects can be included (along with those of the mass term) by simply making the substitution $m \rightarrow m+a \Lambda^{2}$, where $\Lambda$ is required by dimensional analysis. Thus, we are led to the potential (4) with coefficients]

$c_{1} \sim m \Lambda^{3}+a \Lambda^{5}, c_{2} \sim m^{2} \Lambda^{2}+m a \Lambda^{4}+a^{2} \Lambda^{6}$.

We now study the alignment of the condensate, and the consequent pattern of symmetry breaking, as we reduce $m$ at fixed (small) $a$. For $a \Lambda^{2} \ll|m| \ll \Lambda$ the discretization errors in $c_{1}$ and $c_{2}$ are a small correction, and symmetry breaking is as in the continuum. In particular, since $c_{2} / c_{1} \sim|m| / \Lambda \ll 1$, only the first term in the potential is important, and the condensate aligns as $\Sigma_{0}= \pm 1$. Flavor and parity are unbroken.

This situation persists even when $m$ becomes of size $\sim a \Lambda^{2}$. Now discretization errors are important, but $c_{2} / c_{1} \sim a \Lambda$ is still small, and so the condensate is aligned as before. The effect of the discretization errors is to shift the mass at which $c_{1}$ vanishes from $m=0$ to $m^{\prime}=0$, with $m^{\prime}=m-a \Lambda^{2}$ (recall that all constants are being set to unity). In terms of this new mass, the coefficients become

$c_{1} \sim m^{\prime} \Lambda^{3}, \quad c_{2} \sim m^{\prime 2} \Lambda^{2}+m^{\prime} a \Lambda^{4}+a^{2} \Lambda^{6}$.

Finally, when the shifted masses are of $O\left(a^{2}\right)$, i.e. $a m^{\prime} \sim(a \Lambda)^{3}$, the coefficients simplify to

$c_{1} \sim m^{\prime} \Lambda^{3}, \quad c_{2} \sim a^{2} \Lambda^{6}$.

The crucial point is that $c_{1} \sim c_{2}$, and, as we show below, competition between the two terms can lead to spontaneous flavor and parity breaking, i.e. the appearance of an Aoki phase? It follows that the width of the Aoki phase is $\Delta m_{0} \sim a \Delta m^{\prime} \sim(a \Lambda)^{3}$.

Let us now examine the potential (4) in some detail to determine the condensate $\left\langle\Sigma_{0}\right\rangle$. Writing $\Sigma=A+i \mathbf{B} \cdot \boldsymbol{\sigma}$ with $A^{2}+\mathbf{B}^{2}=1$, the potential becomes

$\mathcal{V}_{\chi}=-c_{1} A+c_{2} A^{2}$,

${ }^{7}$ Since we are keeping terms of $O\left(a^{2}\right)$ in $c_{2}$, we must also include such terms in $\mathcal{L}_{\text {eff }}$, Eq. (11). This has been done in 2 and does not change (5).

${ }^{8}$ When the first two terms of the potential are comparable, the cubic $c_{3}$-term is down by a power of $a \Lambda$, except for a small region of order $(a \Lambda)^{4}$ in which $c_{1} \sim c_{2} \sim c_{3}$. 
The parameter $A$ is constrained to lie between -1 and +1 inclusive. Under flavor $S U(2)_{V}$ rotations, $A$ is invariant while $\mathbf{B}$ rotates by an orthogonal transformation. Hence, when the vacuum state $\Sigma_{0}=A_{0}+i \mathbf{B}_{0} \cdot \boldsymbol{\sigma}$ develops a non-zero $\mathbf{B}_{0}$, the flavor symmetry breaks spontaneously to a $U(1)$ subgroup defined by $\exp \left\{i \theta \hat{\mathbf{B}}_{0} \cdot \boldsymbol{\sigma}\right\}$. A non-zero value of $\mathbf{B}_{0}$ can occur only when $\left|A_{0}\right|$ is strictly less than one.

The sign of $c_{2}$, which is not predicted by our analysis, determines two qualitatively different behaviors. We discuss $c_{2}>0$ first, since this gives rise to an Aoki phase. The potential is then a concave upward parabola with absolute minimum at $A_{m}=c_{1} / 2 c_{2} \equiv \epsilon$. If this minimum lies outside the range -1 to 1 , then the vacuum is at $A_{0}= \pm 1$ and flavor symmetry is not spontaneously broken. But if $\left|A_{m}\right|<1$, then the vacuum is at $A_{0}=\epsilon$, and flavor symmetry is spontaneously broken. The system thus finds itself in the Aoki phase with two massless Goldstone pions. The mass spectrum is

$$
\begin{array}{r}
m_{1}^{2}=m_{2}^{2}=0, \frac{m_{3}^{2} f_{\pi}^{2}}{2 c_{2}}=1-\epsilon^{2} \text { for }|\epsilon| \leq 1 \\
\frac{m_{a}^{2} f_{\pi}^{2}}{2 c_{2}}=|\epsilon|-1 \text { for }|\epsilon| \geq 1,
\end{array}
$$

which is illustrated in Fig. 2. Recalling from (7) that $\epsilon \sim m^{\prime} /\left(a^{2} \Lambda^{3}\right)$, we see explicitly that the Aoki phase has a width $\Delta m_{0} \sim a \Delta m^{\prime} \sim(a \Lambda)^{3}$.

The $c_{2}<0$ case gives a concave downward parabola with an absolute maximum at $A_{m}=\epsilon$, and therefore the vacuum is always at $A_{0}= \pm 1$ for any value of $\epsilon$. Flavor symmetry is not spontaneously broken, and there are three degenerate pions with non-zero masses $m_{a}^{2} f_{\pi}^{2} / 2\left|c_{2}\right|=1+|\epsilon|$.

In summary, in the continuum, when the quark mass passes through zero, the condensate jumps discontinuously from $\Sigma_{0}=+1$ to $\Sigma_{0}=-1$, with the pions becoming massless at the transition point. On the lattice, the explicit chiral symmetry breaking due to the Wilson term alters this transition. If $c_{2}>0$, the condensate rotates continuously from +1 to -1 , breaking flavor and parity as it does so. If $c_{2}<0$, the condensate jumps discontinuously as in the continuum, but the pions never become massless. This shows how the

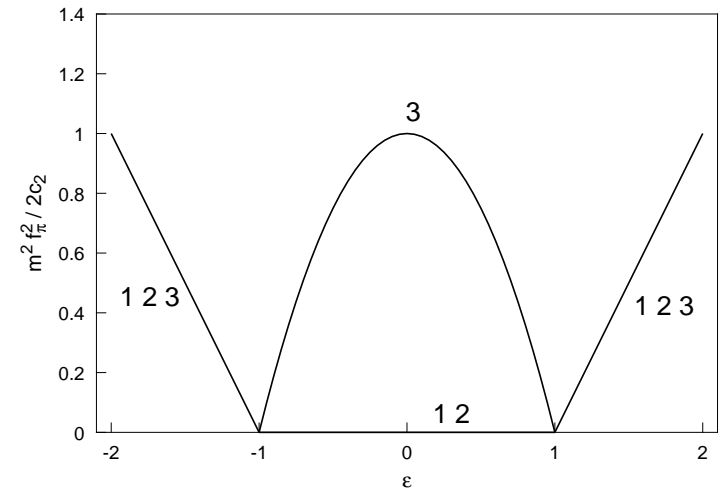

Figure 2. Pion masses as a function of $\epsilon=c_{1} / 2 c_{2}$ for $c_{2}>0$. The curves are labeled by the flavor of the corresponding pion.

Aoki phase is intimately related to chiral symmetry breaking. Our analysis cannot determine the sign of $c_{2}$, and indeed $c_{2}$ can have different signs for different choices of discretization of the Dirac operator. Numerical evidence to date is consistent with $c_{2}>0$ for both unimproved and improved Wilson fermions.

We close with a few comments [2]. First, one can relate the appearance of the Aoki phase to a non-vanishing density of zero modes of the hermitian Wilson-Dirac operator. We stress, however, that our analysis applies only to the unquenched theory, and has no direct implications for the spectrum of the Wilson-Dirac operator in the quenched theory. Second, our analysis applies as well to $O(a)$ improved Wilson fermions. And, finally, we stress that the phase structure will be different for $N_{f}=3$, for which there is no $m \rightarrow-m$ symmetry in the continuum.

\section{REFERENCES}

1. S. Aoki, Phys. Rev. D30 (1984) 2653; Phys. Rev. Lett. 57 (1986) 3136; Prog. Theor. Phys. 122 (1996) 179.

2. S. Sharpe and R. Singleton Jr., Phys. Rev.D58, 074501 (1998).

3. M. Creutz, hep-lat/9608024.

4. K. Symanzik, Nucl.Phys.B226(1983)187,205.

5. M. Lüscher et al.,Nucl.Phys.B478(1996)365. 